\title{
A dieta hipossódica na percepção de indivíduos hipertensos hospitalizados
}

\section{Hyposodic diet in the perception of hospitalized hypertensive individuals}

\author{
Elizabeth Cristina Verrengia ${ }^{1}$ \\ Anete Araúio de Sousa ${ }^{2}$ \\ 1,2 Programa de Pós-Graduação em Nutrição, \\ Núcleo de Pesquisa de Nutrição em Produção de \\ Refeições (NUPPRE). Universidade Federal de \\ Santa Catarina. Florianópolis, SC, Brasil. \\ Correspondência / Correspondence \\ Elizabeth Cristina Verrengia \\ Unidade de Ensino Superior Ingá, Faculdade de \\ Ensino Superior Ingá. \\ Avenida Colombo, 9727 - KM 130 \\ 87070-000 - Maringá, PR, Brasil \\ E-mail: lizverrengia@hotmail.com
}

\section{Resumo}

A pesquisa objetivou conhecer a percepção de indivíduos hipertensos sobre a dieta hipossódica em um hospital público em Florianópolis, Brasil. Trata-se de pesquisa com abordagem qualitativa na qual foram realizadas 15 entrevistas semiestruturadas, aplicadas a pacientes hospitalizados há quatro ou mais dias consumindo dieta hipossódica. O modelo de análise consistiu de duas categorias: consumo da dieta hipossódica e limites para o consumo da dieta hipossódica. O baixo consumo da dieta hipossódica relacionou-se à falta de sabor, ao modo de preparo dos alimentos e a intolerâncias pessoais. Os elementos facilitadores para o consumo alimentar foram: restrição de sal quando já incorporada ao hábito alimentar, concepção de que o excesso de sal é prejudicial à saúde e papel da família na adesão ao tratamento. Concluiu-se que, para melhorar o consumo da dieta hipossódica no ambiente hospitalar, deve-se agregar o aspecto sensorial ao nutricional, atentando para as preferências alimentares dos pacientes.

Palavras-chave: Dieta hipossódica. Serviço Hospitalar de Nutrição hospitalar. Aceitação pelo Paciente de Cuidados de Saúde. Percepção.

\section{Abstract}

This research aimed to determine the perception of hypertensive individuals following a hyposodic diet in a public hospital in the city of Florianopolis, Brazil. This qualitative research was carried 
out through 15 semi-structured interviews applied to patients hospitalized for four or more days and maintained on a hyposodic diet. The model of analysis comprised two categories: following a hyposodic diet and limits for following a hyposodic diet. The low adherence to low hyposodic diet was related to tastelessness, the way meals were prepared, individual intolerance and difficulties in consuming some foods. The facilitating factors for acceptance were: sodium restriction incorporated into the eating habits, assumption that excess salt is bad for health, and the role played by the family in the adherence to treatment. The research concluded that to improve the acceptance of a hyposodic diet in hospital it is necessary to associate the nutritional factors with the sensorial aspects, considering the dietary preferences of patients.

Key words: Sodium-Restricted. Food Service, Hospital. Patient Acceptance of Health Care. Perception.

\section{Introdução}

A hipertensão arterial é um importante desafio para a saúde pública mundial devido a sua alta prevalência e risco concomitante para doenças cardiovasculares e renais. Atualmente, mais de $25 \%$ da população mundial é hipertensa e há estimativas de que este percentual poderá chegar a $29 \%$ até $2025,{ }^{1,2}$

A dieta hipossódica, apesar de citada mundialmente como uma das maneiras mais efetivas de controle na hipertensão arterial, apresenta um dos menores índices de adesão. ${ }^{3,4}$ Esta não-adesão tende a se agravar nas hospitalizações, pois a alimentação hospitalar é conhecida por apresentar características como falta de sabor e baixa temperatura, além de ser percebida como algo proibido ou permitido, dependendo da doença. ${ }^{5}$ A rejeição da dieta hipossódica pode levar a uma ingestão insuficiente, resultando em inadequação de energia e nutrientes no corpo. ${ }^{4,6}$ Apesar das consequências negativas da ingestão insuficiente desta dieta no ambiente hospitalar, pouco se sabe sobre os motivos da não-adesão dos pacientes. Alguns estudos têm destacado que a baixa ingestão alimentar por parte do paciente internado envolve aspectos como palatabilidade das preparações, associada à ausência de sal; ${ }^{4,7-10}$ a temperatura fria de alimentos que deveriam ser servidos quentes; ${ }^{8}$ o horário; ${ }^{810}$ a apresentação da refeição;, ${ }^{7,8}$ a falta de orientação do paciente pela equipe de saúde, a interferência na socialização (dificuldade para alimentar-se fora de casa e conflitos familiares, pelo consumo não permitido de preparações ricas em sódio) e a falta de opções de alimentos industrializados com baixo conteúdo de sódio, gerando limitação nas escolhas alimentares. ${ }^{4,9}$ 
Tendo em vista a alta prevalência da hipertensão arterial, aliada ao baixo consumo da dieta hipossódica, destaca-se a relevância de estudos que busquem compreender os fatores que influenciam o consumo de sódio, pois este é efetivamente consenso no tratamento nãofarmacológico dessa enfermidade. Assim, o objetivo da pesquisa foi conhecer a percepção de indivíduos hipertensos em relação à dieta hipossódica em um hospital público no município de Florianópolis, Estado de Santa Catarina.

\section{Método}

Trata-se de estudo do tipo exploratório com abordagem qualitativa, desenvolvido em um hospital público no município de Florianópolis. A abordagem qualitativa responde a questões que não podem ser quantificadas, pois se aprofunda no mundo dos significados das ações e relações humanas. Além disso, neste tipo de estudo, os resultados podem gerar pressupostos para futuras pesquisas. ${ }^{11}$

Foram entrevistados 15 indivíduos com idade entre 29 e 89 anos, com tempo de internação entre cinco e 35 dias. A coleta de dados foi realizada em setembro de 2007. Dados como idade, sexo, diagnóstico dos pacientes foram obtidos dos prontuários. Esta etapa possibilitou a seleção de pacientes segundo critérios de inclusão e exclusão previstos preliminarmente. Os critérios de inclusão compreenderam: estar há mais de quatro dias hospitalizado e recebendo dieta hipossódica com consistência normal; ter mais de uma hospitalização e assinar o Termo de Consentimento Livre e Esclarecido. Foram excluídos do estudo pacientes disfásicos, inconscientes, confusos ou sedados, gestantes, pacientes em terapia nutricional enteral ou parenteral e em tratamento para doenças renais e hepáticas.

Para os pacientes que atenderam a esses critérios, foi realizado um primeiro contato, interrogando-os sobre a possibilidade de participarem da pesquisa. Para aqueles que respondiam afirmativamente, realizou-se a entrevista no momento mais conveniente para o entrevistado com assinatura do TCLE. Todas as entrevistas foram realizadas ao leito dos pacientes. A duração variou entre 15 a 30 minutos e foram registradas em gravador digital.

A técnica utilizada para a coleta de dados foi a entrevista semiestruturada. O estudo desenvolvido por Poulain ${ }^{12}$ forneceu suporte para a elaboração de um modelo de análise composto por duas categorias: Consumo da dieta hipossódica no hospital e Limites para o consumo da dieta hipossódica no hospital. A partir da pergunta norteadora, "O que o sr.(a) acha das refeições que está recebendo no hospital?" e das respostas obtidas, outras questões foram empregadas com o objetivo de esclarecer ou explorar informações comentadas pelos pacientes, sempre com o cuidado de não se manter afastado do tema central. 
As entrevistas foram realizadas até se alcançar a saturação teórica, ${ }^{13}$ ou seja, quando estas não produziram mais informações novas e responderam ao objetivo da pesquisa, tornando-se desnecessário um critério numérico para garantir sua representatividade ${ }^{11}$ e as relações entre categorias estão bem estabelecidas e validadas. ${ }^{11}$ Para atender ao critério de saturação teórica, as entrevistas foram transcritas diariamente. Depois de transcritos, os relatos foram comparados com a gravação correspondente.

Os dados foram analisados segundo a técnica de análise de conteúdo, na qual se buscou identificar as categorias que respondessem ao objetivo geral da pesquisa. Classificar elementos em categorias impõe identificar o que cada um deles tem em comum com o outro..$^{13}$

Alguns relatos foram transcritos nos resultados e, a fim de assegurar o anonimato dos participantes, foram utilizadas as letras $\mathrm{H}$ (homens) e M (mulheres), seguidas pela idade dos pacientes (Ex: H 48; M 57).

Esta pesquisa foi aprovada pelo Comitê de Ética em Pesquisa com Seres Humanos da Universidade Federal de Santa Catarina, processo número 209/07.

\section{Resultados e discussão}

A análise de conteúdo resultou na sistematização de unidades de significado em categorias que podem auxiliar na compreensão de como pacientes percebem a dieta hipossódica no ambiente hospitalar.

Consumo da dieta hipossódica: controle, apoio familiar e incorporação do hábito alimentar

A partir dos relatos, identificaram-se elementos que podem facilitar o consumo da dieta hipossódica no hospital. Entre eles destaca-se a restrição de sal quando já incorporada ao hábito alimentar, a concepção de que o excesso de sal é prejudicial à saúde e o papel da família na adesão ao tratamento.

"Em casa a comida é controlada também. Porque a tempo eu tenho pressão alta. Então o sal é controlado, a comida minha é controlada." (H 66).

A concepção de que o excesso de sal é prejudicial à saúde pode contribuir para melhorar o consumo da dieta hipossódica. Em alguns relatos, conhecimentos sobre as recomendações desta dieta e sua função terapêutica mostraram-se facilitadores do autocuidado.

"Comer com menos sal é melhor, porque eu estava tomando remédio da pressão em casa porque a gente usa mais sal. E aqui não, não estou tomando e está sempre 10/6, 11/7.” (M 57). 
"Eu acho que eles devem fazer do jeito que a gente pode comer. Na minha casa eu como pouco sal mesmo, minha comida é mais insossa." (M 64).

Apesar da importância do conhecimento na adesão terapêutica, esta informação é muitas vezes negligenciada. Mellen et al., ${ }^{14}$ ao avaliarem a prevalência de aconselhamento nutricional e atividade física para pacientes hipertensos, verificaram que, apesar da clara evidência da eficácia das mudanças de estilo de vida como tratamento primário ou coadjuvante da hipertensão arterial, o número de indivíduos que recebem esse tipo de orientação ainda é baixo. Apenas $35 \%$ dos pesquisados tinham recebido aconselhamento nutricional e $26 \%$, orientados a praticar atividade física.

A família parece ter papel importante na incorporação e manutenção desse hábito alimentar em domicílio.

\section{[...] "ela [a esposa] toma alguns cuidados e prepara a comida já com pouco sal." (H 66).}

A hipertensão arterial provoca limitações no estilo de vida não apenas do hipertenso, mas do seu núcleo familiar. ${ }^{15}$ Portanto, o papel da família na incorporação e manutenção do hábito familiar pode auxiliar na adesão ao tratamento dietético, como evidenciado nos relatos. O apoio familiar é um dos elementos de segurança para os pacientes caminharem para esta nova perspectiva de vida.

Limites para a ingestão da dieta hipossódica: sal e sabor, contexto familiar, forma de preparo dos alimentos e falta de apetite

Embora considerem a alimentação aparentemente boa e compreendam a necessidade de controle, alguns pacientes a rejeitam. Ou ainda, o comer sem sal é sinônimo de obrigação e de falta de sabor:

"Eu estou achando muito bonito [apresentação], mas não tem sabor. Porque é sem sal. É o rei do sabor! Faltou, não é? Se não, não dá! Pra mim só vem um grama de sal, então eu só como aquilo que tem aquele salzinho. Mas mesmo assim se cozinhou sem nada não pega. Então quando dá ali pra enrolar a gente, só mata a fome."(M 69).

Stanga et al., ${ }^{8}$ Yabuta et al..$^{10}$ (2006) e Demário et al. ${ }^{16}$ também identificaram a falta de sabor como causa para a menor aceitação alimentar e, consequentemente, menor ingestão de nutrientes. O sabor da refeição hospitalar é muitas vezes visto como secundário em detrimento da função nutricional e higiênico-sanitária. ${ }^{5}$ Em dietas restritas, como a hipossódica, as preparações tornam-se pouco palatáveis, dificultando a adesão ao tratamento e reduzindo a ingestão calórica dos pacientes. ${ }^{8}$.

Para amenizar a falta de sabor das dietas hipossódicas, algumas estratégias podem ser incorporadas à alimentação hospitalar, entre as quais a personalização das dietas, que visa aproximar a dieta hospitalar do hábito alimentar do paciente. Outra ação é a seleção de alimentos 
com diferentes formas, texturas e cores. O apelo visual pode ser uma maneira de aumentar o consumo alimentar no hospital. ${ }^{17}$ (HESS, 1997). Os condimentos e ervas aromáticas, quando utilizados corretamente, podem potencializar o sabor dos alimentos e serem uma alternativa ao sal. ${ }^{18}$

Ao analisar os aspectos que podem dificultar o consumo da dieta hipossódica, pode-se verificar que a família tanto pode contribuir, como limitar ou impossibilitar este processo.

[..] "O genro, com pouco sal, reclama... Eu moro com a filha, sou viúva. Então o genro se falta sal, já fala: Ei, está faltando sal? Quer que eu vá comprar? Aí a gente tem que botar sal normal. Aí a pressão subia mais.” (M 67).

"Em casa é tudo meio desajeitado porque eu cozinho sozinho, minha mulher trabalha, não é a mesma coisa. Ai eu exagero!” (H 48).

Morar sozinho sem o apoio familiar ou com familiares que não participam da modificação dietética para melhorar a saúde do membro da família pode contribuir para a não-adesão à dieta hipossódica. ${ }^{15}$ Este apoio é reconhecido por contribuir para a aceitação da dieta hipossódica no hospital.

O modo de preparo dos alimentos no hospital parece interferir na ingestão da dieta hipossódica, principalmente quando não se assemelham às práticas alimentares dos pacientes. Neste sentido, são identificados aspectos relacionados ao uso insuficiente de ervas aromáticas para temperar os alimentos e tipo de cocção, especialmente as preparações fritas e assadas, que são preferidas em relação às ensopadas. As queixas surgiram ao longo dos relatos, mas há uma tendência à compreensão e resignação com o serviço hospitalar.

[...] "na casa da gente, eu jogo bastante tempero, jogo bastante erva, tudo que tem direito. E aqui, nós estamos aqui e não dá pra fazer, porque é uma tonelada de gente comendo, não tem condições. Eu acho assim, não tem possibilidade mesmo.” (M 57).

Corbeau ${ }^{19}$ argumenta que os indivíduos hospitalizados possuem preferências e hábitos alimentares diversos, construídos antes da hospitalização. Aprender a respeitar as preferências e hábitos pode ser uma estratégia para a construção, em conjunto com o paciente, de uma relação mais saudável com a alimentação.

Os hábitos e preferências alimentares podem interferir no consumo da dieta hipossódica, pois o tempero de casa remete a recordações, sensações e sabores que as refeições servidas no hospital não fornecem. A alimentação vai além de satisfazer a fome fisiológica, pois há necessidade de satisfazer os apetites, os desejos de experimentar novamente sensações, prazeres e relembrar laços sociais que acompanham o gosto. ${ }^{19,20}$

[...] "em casa tem a qualidade, tem o amor, tem tudo! Tem o carinho... a comida é feita com mais amor "[...]. (H 66). 
Os alimentos não são identificados pelo paciente quando reduzidos a uma regra, norma ou rotina do nutriente. Portanto, a alimentação hospitalar está longe de incorporar outros comeres que não só o comer nutriente e as suas dimensões simbólicas e sensoriais nem sempre são consideradas. ${ }^{19}$

Por outro lado, ao mesmo tempo em que compreendem os limites dos serviços ligados à alimentação hospitalar, há expectativas em relação à refeição, e identificaram-se preferências alimentares. As preferências são semelhantes, no preparo ou na composição, àquelas consumidas em casa. As saladas, as frutas, o arroz e o feijão e a sopa foram os alimentos mais citados.

"Olha aqui o que eu mais gostei foi a sopa. Por que eu sou acostumado, em casa também a noite é a minha sopa." (H 68).

As expectativas também estiverem relacionadas à percepção de que a alimentação hospitalar deve ser nutricionalmente correta e saudável:

"Eu gosto muito de salada e de fruta, podia aumentar porque já que a gente está no hospital, faz bem pra saúde!” (M 71).

No estudo de Watters et al., ${ }^{21}$ investigando as percepções de adultos sobre os serviços de alimentação e nutrição hospitalar através de grupos focais, os pacientes demonstraram que a comida servida em hospitais deveria ser modelo de dieta saudável.

Garcia ${ }^{22}$ argumenta que práticas alimentares originadas de preocupação com a saúde mostramse conflituosas, com comportamento alimentar formado por representações construídas ora pela preocupação com a saúde e ora pelo paladar. Assim, em determinada situação, a alimentação poderá vir acompanhada de culpa, a exemplo dos relatos de pacientes que não adotam a dieta em domicílio.

O alimento também é causador de mal-estar e, portanto, rejeitado. A rejeição esteve relacionada também a sua consistência e, consequentemente, com a impossibilidade de consumi-lo.

"Eu não posso comer ovo por que me ataca o fígado e ontem já veio ovo, aí eu deixo de lado e não como nada. Só comi a salada.” (M 68).

"Maçã, que eu não posso comer [risos], é que eu não tenho dente... As coisas pra mim tem que ser mais maciazinha...” (M 71).

Dados de Pedroso, Sousa \& Salles ${ }^{23}$ em pesquisa utilizando grupos focais com nutricionistas, observaram que a individualização da dieta parece ser um desafio para os profissionais envolvidos com a produção das refeições, em função da padronização do processo. Sousa e Proença ${ }^{5}$ discutem que há dificuldade de se perceber o paciente no processo produtivo, pois o fluxo dos alimentos segue a lógica da padronização e da divisão do trabalho. 
No mesmo sentido, a doença interfere no apetite. Os entrevistados relataram sensações que acabaram inviabilizando a alimentação, apesar de a considerarem atrativa.

"Tudo que vem é bom. Vem saladinha, a gente gosta de comer. Só que é só comer, sente um fastio. Então não como, vai quase tudo embora...” (M 68).

Demário, Sousa e Salles ${ }^{16}$ observaram que a doença alterou o apetite dos pacientes, principalmente quando acompanhada de mal-estar e dor.

Quanto ao horário, apesar de não ser o habitual dos pacientes, referem necessidade de adaptação, por entenderem ser o horário correto, ou ainda, conformam-se com uma realidade que parece imutável.

"É meio cedo, mas o quê que se vai fazer! Tem que encarar!" (H 48).

"Já acostumei! Quase 40 dias que estou aqui!" (M 57).

Na pesquisa de Demário et al., ${ }^{16}$ o horário das refeições hospitalares foi considerado um modelo a ser seguido. Os autores colocam, ainda, que esses resultados podem demonstrar o controle e disciplina envolvidos na alimentação hospitalar.

A noção de necessidade de controle, tão destacada nos relatos, pode revelar, em parte, a concepção de medicalização da alimentação hospitalar. ${ }^{19}$ E ainda, receio de serem mal-interpretados ao se queixarem das condições de cuidado e da alimentação. ${ }^{12}$

\section{Conclusões}

No local de estudo, os indivíduos hipertensos perceberam que a dieta hipossódica apresenta falta de sabor decorrente da ausência de sal no processo de cocção e que o modo de preparo difere dos hábitos da alimentação em casa. Outros elementos apontados foram falta de apoio familiar, falta de apetite provocada pela doença e preferências e intolerâncias individuais a alguns alimentos. Portanto, agregar o aspecto sensorial ao nutricional, atentando para as preferências alimentares dos pacientes, pode melhorar o consumo da dieta hipossódica durante a hospitalização.

Ressalta-se que negligenciar o sabor no preparo da dieta hipossódica é um problema frequente na alimentação hospitalar, e que a adição de condimentos, ervas aromáticas e sal de forma controlada na cocção pode melhorar o sabor das preparações.

O conhecimento e a compreensão desses aspectos pela equipe de saúde podem transformar a alimentação hospitalar em uma experiência que conjugue aspectos sensoriais e nutricionais. 


\section{Referências}

1. Kearney P, Whelton M, Reynolds K et al. Global burden of hypertension: analysis of worldwide data. The Lancet. 2005;(365):217-23.

2. Hypertension: uncontrolled and conquering the world. The Lancet. 2007;18(370):539.

3. Mion JRD, Pierin AMG, Guimarães A. Tratamento da hipertensão arterial: respostas de médicos brasileiros a um inquérito. Rev Ass Med Bras. 2001;3(47):249-54.

4. Bentley B et al. Factors related to non-adherence to low sodium diet recommendations in heart failure patients. Eur J Cardiovasc Nurser. 2005;(4):331-6.

5. Sousa AA, Proença RPC. La gestion des soins nutritionnels dans lê secteur hospitalier: une etude comparative Bresil-France. Rech Soins Infirm. 2005(83):28-33.

6. Dupertuis YM, Kossovisky MP, Kyle UG et al. Food intake in 1707 hospitalized patients: a prospective comprehensive hospital survey. Clin Nutr. 2002;(22):115-23.

7. Neily JB, Toto KH, Gardner EB et al. Potential contributing factors to noncompliance with dietary sodium restriction in patients with heart failure. Am Heart J. 2002;(143):29-33.

8. Stanga Z, Zurfluh Y, Roselli M et al. Hospital food: a survey of patients' perceptions. Clin Nutr. 2003;3(23):241-6.

9. Prieto DB, Leandro-Merhi VA, Mônaco DV et al. Intervenção nutricional de rotina em pacientes de um hospital privado. Rev Bras Nutr Clin. 2006;3(21):181-7.

10. Yabuta YC, Cardoso E, Isosaki M. Dieta hipossódica: aceitação por pacientes internados em hospital especializado em cardiologia. Rev Bras Nutr Clin. 2006;1(21):33-7.

11. Minayo MCS, Deslandes SF, Cruz Neto O. Pesquisa social: teoria, método e criatividade. 24a ed. Petrópolis: Vozes; 2004.

12. Poulain JP. Etat des lieux: Comité de Liaison Alimentation Nutrition. In: Ricour C. Mise em place d'une politique nutritionnelle dans lês etablissements de santé. Paris: Ministère de la Santé; 2002.

13. Bardin L. Análise de conteúdo. 3a ed. Lisboa: Edições 70; 2004.

14. Mellen PB, Palla SL, Goff DC Jr, Bonds DE.J Gen Intern Med. 2004 Sep;19(9):917-24

15. Araújo TL, Maciel ICF, Maciel GGF et al. Reflexo da hipertensão arterial no sistema familiar. Rev Soc Cardiol. 1998;2(8):1-6.

16. Demario RL, Sousa AA, Salles RK. Comida de hospital: percepções de pacientes em um hospital público com proposta de atendimento humanizado. Ciênc saúde colet. 2010;(15):1275-82.

17. Hess MA. Taste: the neglected nutricional factor. J Am Diet Assoc. 1997;10(97):205-7.

18. Bentley B, Moser DK. Dietary sodium in heart failure: what to tell your patients. Prog Cardiovasc Nurs. 2007;1(22):41-2.

19. Corbeau JP. Alimentar-se no Hospital: as dimensões ocultas da comensalidade. In: Canesqui MG, Garcia RWD, Organizadoras. Antropologia e nutrição: um diálogo possível. Rio de Janeiro: Fiocruz; 2005. 
20. Pilla MCBA. Gosto e deleite: construção e sentido de um menu elegante. Hist Quest Deb. 2005;(42):53-69.

21. Watters CA, Sorensen J, Fiala A et al. Exploring patient satisfaction with foodservice through focus groups and meal rounds. J Am Diet Assoc. 2003;10(103):1347-9.

22. Garcia RWD. Alimentação e saúde nas representações e práticas alimentares do comensal urbano. In: Canesqui MG, Garcia RWD, Organizadoras. Antropologia e nutrição: um diálogo possível. Rio de Janeiro: Fiocruz; 2005. p. 211-26.

23. Pedroso CGT, Sousa AA, Salles RK. Cuidado nutricional hospitalar: percepção de nutricionistas para atendimento humanizado. Ciênc saúde colet. 2011;(16 supl.1):1155-62.

Recebido: 26/2/2012

Aprovado: $18 / 9 / 2012$ 